\title{
Patient-held Logbooks for Cancer Care Treatment: the Users' Evaluative Perspective
}

\author{
Quynh Lề ${ }^{1}$, Stuart Auckland ${ }^{1}$, Hoang Boi Nguyen ${ }^{1}$, Daniel R Terry ${ }^{1, *}$, Dustin V.A. Lê ${ }^{2}$ \\ ${ }^{1}$ University Department of Rural Health, University of Tasmania, Launceston, 7250, Tasmania, Australia \\ ${ }^{2}$ Faculty of Medicine, Nursing and Health Sciences, Clayton campus, Monash University, 3800, Victoria, Australia \\ * Corresponding Author: Daniel.Terry@utas.edu.au
}

Copyright (C) 2013 Horizon Research Publishing All rights reserved.

\begin{abstract}
The quality and usefulness of patient held logbooks as a record to improve communication between healthcare professionals providing cancer care was evaluated. Its aim was to facilitate greater coordination and information sharing between patients, carers, clinicians and other health professionals. A mixed-method exploratory study conducted 12-week post distribution of logbooks to patients undertaking cancer care. Data was gathered through questionnaires returned by 66 participants (response rate of $57.4 \%$ ) and interviews with five clinical oncology nurses at a Tasmanian hospital. The logbook quality was evaluated against two sets of indicators, including content and layout. The general effectiveness of the patient logbook was also assessed based on its usefulness, usability, efficiency and satisfaction. The logbook was considered useful among 63 (95.1\%) participants. They acknowledged the logbook enabled them to be better informed, created a feeling of empowerment and greater control over their medical condition. The logbook was observed to improve communication between clinicians, their patients and families. The evaluation generated rich, in-depth information and provided useful insights into the general quality and usefulness of the logbook for cancer care. The strengths and weaknesses of the logbook were highlighted and how to better utilise its functions in the current medical system.
\end{abstract}

Keywords atient-Held Medical Record, Empowerment, Logbook, Communication, Medical Information, Cancer Care

\section{Introduction}

The care of those experiencing cancer is complex and at times involves many health care professionals from oncologists, general practitioners to registered nurses, social workers and many other service providers within the hospital and community settings. [1] So too, the process is complex and has been shown to be a confusing and challenging time for both patient and family as they traverse the disease and care experience. [2,3] It has been highlighted that throughout this time communication has a propensity to be less formalised, care to be fragmented and it can be a less smooth process. [1] This has the potential to impact on patient care and meeting their physical and emotional needs. To overcome many of these challenges, the implementation of patient-held medical records or patient logbooks has increasingly been used widely within the health care setting. [1]

Patient-held medical records have been used for chronic disease management, mental health, maternity and child health and the treatment of cancer. [1,4-6] In many cases, patient-held medical records have been shown to facilitate the provision and continuity of care and empowered patients to take ownership of their disease and subsequent treatment [4-11] Patient-held records have also been shown to improve communication between patients and their families; patients and clinicians. Furthermore, they can facilitate the discussion regarding end-of-life care. $[1,5,12]$

Many studies have shown, when appropriately developed, designed and utilised; patient-held records are observed to increase user involvement with greater engagement and cooperation between health care staff and patients. [5,7-9,13] Ritchie [4] states more effective communication between health care staff and patients may also result in improved patient confidence and satisfaction with the care received. In addition, patient-held records have been evaluated and shown to aid clinical governance by evaluating the quality and appropriateness of care provided to patients. [14]

Despite the reported positives of the patient-held medical record, it has been shown that improved clinical outcomes are not always apparent. [7,15] The success of administering patient-held records and their adoption is dependent on the quality and comprehensibility of the records themselves, the attitudes of patients and clinical professionals, and the way they are utilised. [12,16-18] High rates of non-use, incorrect information and unreadable handwriting are all reported to be attributable to the lack of positive outcomes from patient-held records at many 
clinical centres and hospitals. [5,7-9]

As a result, maintaining high quality patient-held records and optimising their usefulness continue to be important elements of good clinical practice. [14] This is particularly imperative when used within cancer treatment, where communication between patients and oncologists may be improved using a mutual decision-making approach. Through this process patients have full access to credible and timely information throughout the cancer journey. [10] In addition, as the cancer treatment dictates the involvement of various practitioners and services, the patient-held record becomes vital to ensure complete and coordinated care remains seamless across the service continuum. $[1,15,19]$

\section{Objectives}

In this context the Cancer Care Network (CanNET) within the Tasmanian Department of Health and Human Services (DHHS) developed a patient-held medical record or logbook. The logbook was developed as a means of recording all relevant data, to enhance the accessibility of information available to patients, and to improve communication between healthcare professionals providing cancer care. Its aim was to facilitate greater coordination and information sharing between patients, carers, clinicians and other health professionals. Within the patient-held record, test results, various appointments, medications received and administered and information concerning the various services was provided and recorded.

As part of the ongoing development of the logbook, and prior to its full implementation, an exploratory evaluation of the logbook was undertaken. The evaluation aimed to appraise the overall quality and usefulness of the patient logbook in terms of being a record of treatment and to streamline the transfer of medical information between specialists and other health professionals. Many studies concerning the use of logbooks have been similarly evaluated for their effectiveness and weaknesses within cancer care. This is particularly evident among the non-experimental studies which provide a greater understanding of the impact logbooks have in patient care. [1] Overall the aims of the study shadowed the future directions provided within the literature which is to better "understand the conditions in which the patient-held record can be used most effectively." [1]

\section{Materials and Methods}

The exploratory research project was based on a mixed methods approach, which is "a procedure for collecting, analysing, and 'mixing' or integrating both quantitative and qualitative data at some stage of the research process within a single study for the purpose of gaining a better understanding of the research problem". [20] By using both quantitative and qualitative data collection techniques, this mixed method approach enabled the acquisition of an in-depth understanding, which was necessary to adequately address the aims of the project.

A survey questionnaire was newly developed to gather quantitative data, in close consultation with health researchers, academics and was informed by the literature in the field of research methodology. As such, it had undergone both face and content validation prior to being implemented. The questionnaire had 19 items, with 18 closed-ended questions being scaled into two groups. The first seven items concerned patient's demographics. The remaining 12 items pertained to the evaluation of the patient $\log$ book in terms of its general quality and usefulness. Lastly, an open ended item within the questionnaire provided a means to capture additional respondent data, including concerns and recommendations, which were unable to be gathered elsewhere within the questionnaire.

The questionnaires were distributed to 115 patients, who had been using the logbooks for 12 weeks with 66 (response rate of 57.4\%) questionnaires being returned. Each participant was advised in writing concerning the scope and purpose of the questionnaire and formal consent to participate was sought and given. All questionnaire data was checked for integrity, coded and entered into Statistical Packages for Social Science (SPSS) version 19. In addition, the open-ended data from the questionnaire was coded and entered into Statistical Packages for NVivo version 9 and checked for data integrity. [21,22]

Table 1. Characteristics of survey patients

\begin{tabular}{|l|l|l|}
\hline & $\begin{array}{l}\text { Number of } \\
\text { participants (n/N) }\end{array}$ & Percentage \\
\hline Gender & & \\
\hline Male & $23 / 65$ & 35.4 \\
\hline Female & $42 / 65$ & 64.6 \\
\hline Age groups & & \\
\hline Under 30 years of age & $2 / 65$ & 3.1 \\
\hline 30 to 40 years of age & $2 / 65$ & 3.1 \\
\hline 41 to 50 years of age & $6 / 65$ & 9.2 \\
\hline 51 to 60 years of age & $16 / 65$ & 24.6 \\
\hline 61 years of age or over & $39 / 65$ & 60.0 \\
\hline $\begin{array}{l}\text { Aboriginal and Torres } \\
\text { Strait Islander background }\end{array}$ & & \\
\hline No & $64 / 64$ & 100.0 \\
\hline Yes & $0 / 64$ & 0.0 \\
\hline English proficiency & & \\
\hline Very Poor & $0 / 64$ & 0.0 \\
\hline Poor & $0 / 64$ & 0.0 \\
\hline Just fine & $3 / 64$ & 4.7 \\
\hline Good & $19 / 64$ & 29.7 \\
\hline Very good & $42 / 64$ & 65.6 \\
\hline Computer ownership & & \\
\hline No & $22 / 63$ & 34.9 \\
\hline Yes & $41 / 63$ & 65.1 \\
\hline & &
\end{tabular}

Qualitative data was collected via face-to-face interviews. These were conducted with five clinical oncology nurses, who had assisted with the distribution of the log books to patients and who had used the logbooks themselves. Each interview took approximately 15-20 minutes and was audio recorded with the participants' consent. The interviews 
were transcribed, coded and also entered into NVivo version 9. [21] The important points and issues emerging from the interview data were identified and discussed with reference to the questionnaire data analysis.

Ethical approval for the study was obtained by the Human Research Ethics Committee (Tasmania) Network prior to commencing the study.

\section{Results}

The results obtained from the questionnaires and interviews provide insights not only into the quality and usefulness of the log books, but also the attitudes and needs of the targeted patients. It must be noted that in some instances participants did not answer some specific questions. Selected characteristics of the targeted patients are presented in Table 1. The results of the evaluation are presented in terms of two key aspects: General quality of the logbook and its functional usefulness.

\subsection{General Quality of the logbook}

The general quality of the logbook was evaluated against two sets of indicators, being content and layout. The content indicators included familiarity, comprehensibility, clarity, and coverage. The layout indicators were ease of reading, ease of finding information and attractiveness. The general finding was that the results were fairly favourable across most of the indicators.

An important finding was that a majority 58 (89\%) of the participants have not used a logbook previously. However, most $63(95.1 \%)$ found the logbook comprehensible. It was indicated by $50(76.2 \%)$ that they had received sufficient instruction how to use the logbook while $15(23.0 \%)$ indicated they had not been instructed on what to do and how to use the logbook and as a result experienced a level of confusion.

The follow-up comments to the open-ended question added further insights into this issue. A patient participant noted,

[The] main problem was lack of information (verbal) on just how to use the log book... When I tried to use mine, the doctor didn't know about them so that was very off-putting for me. I expected some help with filling in details but didn't get it. (Questionnaire Participant)

This lack of an introductory briefing and instruction for patient participants could be partly explained by the time constraints and the small scope of the pilot project. However, it demonstrates the importance of information and awareness raising in any initiative of change.

The information coverage within the logbook was acceptable among $51(76.9 \%)$ of the participants, while the remaining $15(23.0 \%)$ anticipated seeing additional information including diagnosis, surgery requirements and treatment plans. In addition, greater explanation of medical terms such as haemoglobin, white blood cell counts, platelets, and how these are relevant chemotherapy treatments was seen as an important addition. One interview participant discussed what needed to be included for cancer care providers when stating "Probably the diagnosis and surgery and treatment plan would be the main ones. That would be useful for the general practitioner... as correspondence can be long. Quite often the patients go the GP and they say they haven't heard anything." (Interview participant 1)

The majority of participants were positive about the logbook graphics, labelling and layout, including easy-to-read font size, appropriate colour patterns, relevant graphics/images, logical organisation, easy-to-locate information, and attractive design, ranging from 42 (63.5\%) to $56(84.9 \%)$. Positive comments on the layout were found in the open-ended responses. For example, "It [the logbook] puts all the names of doctors, specialists, and medical information I need in one place." (Questionnaire Participant)

Suggestions for the improvement of the logbook include additional space, and labelled tags or colour codes for different sections of the logbook. In addition, some questionnaire and interview participants felt the logbook required greater space and greater room for longer term cancer care. On this issue, one questionnaire participant stated:

My only suggestion is a few more pages are needed. Mainly questions for doctors or specialists although I would continue to use a note pad as the book's pages will be quickly filled. There is so much information in it and stuff I have gathered for it; I would not want to just start another one... I find the book invaluable. (Questionnaire Participant)

Another interview participant stated the patient logbook could incorporate the information in the current system used at the hospital, such as "when their blood is done, size of needles, blood type etc." (Interview participant 3 ) or include "an emergency page on the front of the logbook." (Interview participant 2)

\subsection{Usefulness of the logbook}

The majority of participants found the logbook simple to use $50(76.8 \%)$ and handy to keep $49(76.0 \%)$. Its pocket-size design was highly appreciated for its convenience. Most participants rated the sections on Personal information, Emergency contacts, Information about support centres and the Glossary from 'slightly useful' to 'extremely useful' to them. Other sections regarding the information about Cancer, Test records, Appointment, Chemotherapy information, Test results and Your own comments were also found highly useful to the majority, ranging from $56(84.8 \%)$ to $65(98.1 \%)$ of the participants. Commenting on the function of the logbook as a personal record of treatment, a participant noted, "As I have a chronic disease, this log book has allowed me to keep all my information in one useful place." (Questionnaire participant)

The interaction between patients and their clinicians were found to be enhanced with the use of the logbook. Nearly half, $27(46.6 \%)$, of the participants reported having 
communicated with their GPs or carers through the logbook. It was also found to streamline the transfer of medical information. For example, one participant stated "I find the pages with the results to be very useful when visiting my assorted doctors. I am being treated [in Tasmania] and also... in Melbourne and the communication between them is less than ideal." (Questionnaire participant)

In addition, another participant stated "Overall, the log book is an invaluable aid to persons such as myself, especially as it is specific to my on-going medical interactions with health professionals involved in my journey" (Questionnaire participant). This finding is supported within the literature, which highlights the role patient logbooks are to ensure a seamless and complete care across the service continuum when cancer treatment necessitates the involvement of various practitioners and services. [19]

In addition, the logbooks were observed to actively engage all health professionals while having less impact on the psychological wellbeing of patients. One interview participant stated the logbook was used as a shared medical record "without putting the patient under pressure when they are trying to deal their own illness; it's difficult to rely on the patients to ask them how you are. What treatment are you on?" (Interview participant 5). Nevertheless, as an add-on to the current system, it was noted some sections of the logbook duplicated other available resources or services. This made it less significant in terms of functionality and impact.

A small number of participants reported to use the logbook as frequently as $2-3$ times a week and once per week, $6(10.5 \%)$ and $10(17.5 \%)$ respectively. The highest percentage of participants, $21(36.8 \%)$ used it once per month and $5(8.8 \%)$ of the participants $2-3$ times per year. However, 15 (26.3\%) of the patient participants reported not using the patient logbook at all during the whole pilot project.

More than half of the patients were satisfied with the logbook. In addition $26(52.0 \%)$ of participants acknowledged the role of the logbook in keeping patients better informed, while $28(54.9 \%)$ felt the logbook gave them more control of their medical records and more control over their medical condition. One questionnaire participant highlighted how the logbook empowered them stating

Having the logbook makes me feel more in control and also being more assertive in questioning medical personnel about what blood tests are for and what they are looking for with the different scans I am sent for... Being pro-active about my condition is a real positive of the log book. (Questionnaire Participant)

\section{Discussion and Conclusion}

The findings from the questionnaire and interviews provide implications for the introduction of the patient logbook into the clinical cancer care setting. Valuable and practical recommendations on how to improve the outcomes were also obtained. These recommendations were associated with how to enhance the general quality and usefulness of the patient logbook, including how to regulate its implementation.

As such, this encompassed content such as diagnosis, surgery and treatment plan; more medical terms and their definitions in the Glossary section; and layout including space allocations for each section, labelled tags for different sections of the logbook to make it more useable and efficient. Moreover, greater support needs to be provided, such as providing targeted patient users with more information about how to use the logbook, and how it can be utilised to support their medical journey.

Additional measures included ensuring that healthcare staff are aware and supportive of the implementation of the logbook. Consistency and uniformity of logbook use is a necessity to embed into the culture of the clinics. In addition, collaboration among patients and healthcare professionals should be promoted to ensure a smooth transition to the logbook's adoption without overloading all users. Towards this goal, it is imperative that efforts be oriented towards building an implementation framework with specifications on step by step approaches to the integration of the logbook.

The pilot project produced rich and in-depth information that provided useful insights into the general quality and usefulness of the logbook within cancer care. The strengths and weaknesses of the logbook were highlighted, as well as the suggestions on how to better utilise its functions in the current medical system. Findings from the evaluation provide the first point of reference for the future implementation of the logbook, either at cancer care clinics or within other healthcare sectors. As a whole, the findings signalled a positive response toward the introduction of the logbook. This response is expected to translate into a more active and dedicated commitment towards its adoption in the longer term. It is worth noting that this evaluation was undertaken on a small sample of participants and within tight time constraints, which diminishes the generalisability of the findings.

A logbook has traditionally been used as a tool for keeping track of actions over a period of time. However, it is not just a tool. It can also be a source for establishing bridges to overcome information management divides. It can be an important part of an informative and collaborative health environment. In addition, with the rapid development of electronic technologies, electronic logbooks may also become a powerful source for enhancing health care.

\section{Acknowledgements}

The project was funded by the Cancer Care Network (CanNET) which facilitated the distribution and collection of clients' and service providers' information. 
The authors are profoundly grateful to all the participants who kindly gave their time to respond to the survey and participated in the interviews and the support provided to the researchers by members of the CanNET Team.

\section{REFERENCES}

[1] Gysels M, Richardson A, Higginson IJ. Does the patient-held record improve continuity and related outcomes in cancer care: a systematic review. Health Expectations. 2007;10:75-91.

[2] Sales E. Psychosocial Impact of the Phase of Cancer on the Family. Journal of Psychosocial Oncology. 1992;9:1-18.

[3] Muzzin LJ, Anderson NJ, Figueredo AT, Gudelis SO. The experience of cancer. Social Science \& Medicine. 1994;38:1201-8.

[4] Ritchie L. Evaluation of a patient-held record for Meticillin Resistant Staphylococcus Aureus (MRSA). British Journal of Infection Control. 2007;8:25-9.

[5] Laugharne R, Henderson C. Medical records: Patient-held records in mental health. The Psychiatrist. 2004;28:51-2.

[6] Ko H, Turner T, Jones C, Hill C. Patient-held medical records for patients with chronic disease: a systematic review. Quality and Safety in Health Care. 2010;19:e41.

[7] Cornbleet MA, Campbell P, Murray S, Stevenson M, Bond $\mathrm{S}$. Patient-held records in cancer and palliative care: a randomized, prospective trial. Palliat Med. 2002;16:205-12.

[8] Drury M, Yudkin P, Harcourt J, Fitzpatrick R, Jones L, Alcock C, et al. Patients with cancer holding their own records: a randomised controlled trial. $\mathrm{Br} \mathrm{J}$ Gen Pract. 2000;50:105.

[9] Lecouturier J, Crack L, Mannix K, Hall R, Bond S. Evaluation of a patient-held record for patients with cancer. European Journal of Cancer Care. 2002;11:114-21.

[10] Street AF, Horey D. The State of the Science: Informing choices across the cancer journey with public health mechanisms and decision processes. Acta Oncol. 2010;49:144-52.
[11] Fisher B, Britten N. Patient access to records: expectations of hospital doctors and experiences of cancer patients. Br J Gen Pract. 1993;43:52.

[12] Komura K, Yamagishi A, Akizuki N, Kawagoe S, Kato M, Morita T, et al. Patient-perceived usefulness and practical obstacles of patient-held records for cancer patients in Japan: OPTIM study. Palliat Med. 2013;27:179-84.

[13] Detmer D, Bloomrosen M, Raymond B, Tang P. Integrated personal health records: transformative tools for consumer-centric care. BMC Medical informatics and decision making. 2008;8:45.

[14] Huston JL. The need for mandatory clinical recording standards. J R Coll Physicians Lond. 2004;4:255-7.

[15] Williams J, Cheung W, Chetwynd N, Cohen D, El-Sharkawi S, Finlay I, et al. Pragmatic randomised trial to evaluate the use of patient held records for the continuing care of patients with cancer. Quality in health care. 2001;10:159-65.

[16] Kaelber DC, Jha AK, Johnston D, Middleton B, Bates DW. A research agenda for personal health records (PHRs). JAMIA. 2008;15:729-36.

[17] Keselman A, Slaughter L, Arnott-Smith C, Kim H, Divita G, Browne A, et al., editors. Towards consumer-friendly PHRs: patients' experience with reviewing their health records. AMIA Annual Symposium Proceedings; 2007: American Medical Informatics Association.

[18] Tang PC, Ash JS, Bates DW, Overhage JM, Sands DZ. Personal Health Records: Definitions, Benefits, and Strategies for Overcoming Barriers to Adoption. JAMIA. 2006;13:121-6.

[19] Pascoe SW, Neal RD, Allgar VL, Selby PJ, Wright EP. Psychosocial care for cancer patients in primary care? Recognition of opportunities for cancer care. Fam Pract. 2004;21:437-42.

[20] Ivankova NV, Creswell JW, Stick SL. Using mixed-methods sequential explanatory design: From theory to practice. Field Methods. 2006;18:3.

[21] QRS International. Nvivo 9.0. Melbourne: QRS International Pty Ltd; 2010; Available from: http://www.qsrinternational.c om/\#tab you.

[22] IBM. SPSS Predictive Analytics Software. Armonk, New York 2012 [cited 20118 September]; Available from: http://www-01.ibm.com/software/au/analytics/spss/. 